\title{
Patterns of skin disease in a sample of the federal prison population: a retrospective chart review
}

\author{
Geneviève Gavigan MASc MD, Alana McEvoy MD, James Walker MD
}

Abstract

Background: Dermatology in vulnerable populations is under-researched. Our objective was to analyze the most commonly referred skin diseases affecting the Correctional Service Canada inmates in Ontario.

Methods: An observational, cross-sectional, retrospective chart review of inmate patients seen from 2008 until 2013 was performed. Two groups of patients were included in the analysis: those assessed in-person, and those evaluated by e-consult.

Results: In the in-person patient group, the 3 most common diagnoses were acne, psoriasis and other superficial mycoses. For the e-consult group, the 3 most frequent diagnoses were acne, psoriasis and rosacea. There was a clear bias toward more inmates being seen in-person where the service was provided (Collins Bay Institution) than from other correctional institutions in Eastern Ontario.

Interpretation: Most of the skin diseases that affected the incarcerated population studied were common afflictions, similar to those affecting the general population, which is in agreement with other studies. Future studies investigating skin diseases in male and female inmates across Canada would bestow more generalizable data.

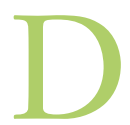

ermatology in vulnerable populations is underresearched. When focusing on the incarcerated population, few studies have described skin disease in inmates. Previous studies have concluded that the skin diseases affecting the inmate population are similar to those affecting the general population. ${ }^{1-7}$ Furthermore, some studies have postulated specific factors in the prison environment that may contribute to skin disease in this population, including stress, ${ }^{1}$ smoking, ${ }^{1}$ substance addiction, ${ }^{5}$ and personal hygiene and overcrowding. ${ }^{2,3,5,7}$ In addition, providing dermatologic treatment within the constraints of the correctional facility may have challenges, such as limitations to the dosing frequency of pills and the dispensing of topical compounds, or the routine use of harsh soaps and skin care products. ${ }^{7}$

The objective of this study was to investigate the most common skin diseases affecting male inmates in Correctional Service Canada institutions in Eastern Ontario and to compare the results with those of previous studies.

\section{Methods}

\section{Setting}

Starting in May 2008, 6 dermatology clinics per year were conducted at Collins Bay Institution (Kingston, Ont.). Consultation requests were placed by referring prison physicians. Patients were escorted and transported to and seen at Collins
Bay Institution from any of the following Eastern Ontario institutions to attend their dermatology appointment: the former Kingston Penitentiary, Bath Institution, Millhaven Institution, Pittsburgh Institution, Frontenac Institution, Regional Treatment Centre and Joyceville Institution. Inmates at Collins Bay Institution were escorted to their appointments, but there was no transport of prisoners beyond the prison confines. Appendix 1 (available at www.cmajopen.ca/content/4/2/ E326/suppl/DC1) summarizes the facility characteristics of the institutions involved; the capacities listed approximate those at the time of consultation. ${ }^{8,9}$

An additional subset of patients was evaluated by e-consult from the non-Collins Bay Institutions, in addition to the more distant Warkworth Institution (Campbellford, Ont.). With e-consult, the dermatologist examined clinical photographs of patients that accompanied a history provided by the referring

Competing interests: James Walker was contracted by Correctional Service Canada during the study time frame as a consultant dermatologist. No other competing interests were declared.

This article has been peer reviewed.

Correspondence to: James Walker, jawalkr@toh.on.ca

CMAJ Open 2016. DOI:10.9778/cmajo.20150135 
physician, which were sent over a secure internet connection. The dermatologist then provided written descriptions and diagnostic and therapeutic instructions to the referring physician. There was no direct interaction between the dermatologist and the patient, and there was no transport of inmates outside of their host institutions.

In Canada, essential physician services are paid for by governments (federal inmates are insured by the federal government), therefore patients evaluated both in person and by e-consult had access to care free of charge.

\section{Design}

Ethics approval was obtained from both the Ottawa Hospital Research Ethics Board and Correctional Service Canada. The charts of all patients who had an in-person dermatology or e-consult assessment while they were inmates at a federal correctional facility between May 1, 2008, and June 30, 2013, were reviewed for this observational, cross-sectional study. The study population was limited to men 18 years of age or older, who had a sentence of 2 years or longer, because the correctional facilities included in this study exclusively housed this demographic. It is noteworthy that all patients underwent evaluation by a single dermatologist, and no other dermatology consultation service was provided to this population during this period.

\section{Analysis}

For every patient, each new diagnosis was recorded. Because there may be less certainty in diagnoses made through e-consult, the in-person and the e-consult groups were analyzed separately to determine the most frequent dermatologic conditions. When a patient underwent both in-person and e-consult evaluation for the same diagnosis, the diagnosis was only recorded for the first encounter. However, when a patient underwent both in-person and e-consult evaluation for different diagnoses, each diagnosis was recorded separately. To assess the most frequent dermatologic conditions, diagnoses were assigned based on the International Statistical Classification of Diseases and Related Health Problems, 10th revision (ICD10). The 5 most common diagnostic groupings were reported for each population, as well as the 3 most common specific diagnoses (based on ICD-10 subheadings).

\section{Results}

A total of 320 patients underwent assessment during the study period (Table 1), 258 of whom were seen exclusively in-person, 60 of whom underwent evaluation exclusively through e-consult, and 2 of whom underwent assessment in both settings with different diagnoses made in each encounter. Each patient received an mean 1.5 (range 0-6) dermatologic diagnoses, and was seen a mean 1.5 (range 1-16) times.

In the in-person consult group, a total of 374 dermatologic diagnoses were provided; 77 diagnoses were given to patients in the e-consult group (Table 2). In the in-person group, the 5 most frequent diagnostic groupings were disorders of skin appendages (88), other disorders of the skin and subcutaneous tissue (57), dermatitis and eczema (56), papulosquamous disorders (54) and mycoses (27). In the e-consult group, disorders of skin appendages (25), dermatitis and eczema (12), papulosquamous disorders (10) and other disorders of the skin and subcutaneous tissue (10), and benign neoplasms (4) were the 5 most commonly encountered diagnostic groupings.

With respect to the specific dermatologic diagnoses, acne (59), psoriasis (45) and other superficial mycoses (17) were the 3 most common diagnoses in the in-person group. Acne (11), psoriasis (6) and rosacea (5) were the 3 most common diagnoses in the e-consult group. Table 2 outlines the frequency of the grouped and specific dermatologic diagnoses.

Of the 258 in-person consults, 117 (45.3\%) of the patients were from the Collins Bay Institution; however, this institution housed only about $225(7.6 \%)$ of the 2965 inmates in Eastern Ontario federal prisons.

Table 1: Patient characteristics

\begin{tabular}{|lc|}
\hline Characteristic & $\begin{array}{c}\text { No. }(\%)^{*} \\
n=320\end{array}$ \\
\hline Patients seen only in person & $258(80.6)$ \\
\hline Patients seen only in e-consult & $60(18.8)$ \\
\hline $\begin{array}{l}\text { Patients seen in both in-person and } \\
\text { e-consult (for different diagnoses) }\end{array}$ & $2(0.6)$
\end{tabular}

Age, yr

Mean age 38. 8 (Range 20-89)

Median 36

Mode 30

Skin phototype

$1-3 \quad 150(46.9)$

4-6 $97(30.3)$

Not recorded $73(22.8)$

Institution

Kingston Penitentiary 17 (5.3)

Collins Bay Institution $\quad 117 \dagger(36.6)$

Bath Institution 34 (10.6)

Millhaven Institution $\quad 30$ (9.4)

Pittsburgh Institution 15 (4.7)

Frontenac Institution $33(10.3)$

Joyceville Institution 27 (8.4)

Regional Treatment Centre $2(0.6)$

Warkworth Institution $\quad 40(12.5)$

Not recorded 5 (1.6)

No. of encounters per patient, mean $1.5(1-16)$ (range)

No. of diagnoses per patient, mean (range)

*Unless otherwise specified.

†All in-person consults. 


\section{OPEN}

Research

\section{Interpretation}

The 4 most common diagnoses seen in our study population were disorders of skin appendages, other disorders of the skin and subcutaneous tissue, dermatitis and eczema, and papulosquamous disorders. With respect to the specific diagnoses, acne and psoriasis were the 2 most frequent diagnoses both in participants who presented in person and those whose condition was diagnosed via e-consult.

Previous studies have found that prisoners are mostly affected by common skin diseases and skin infections. ${ }^{1-7}$ Our results support these conclusions. When comparing our

Table 2 (part 1 of 2): Dermatologic diagnoses

Diagnosis

In person

E-consult

Infections of the skin and subcutaneous tissue

Impetigo

Cutaneous abscess, furuncle, carbuncle

Other local infections of skin and subcutaneous tissue

Bullous disorders

Other bullous disorders

Dermatitis and eczema

Atopic dermatitis

Seborrheic dermatitis

Allergic contact dermatitis

Dermatitis due to substances taken internally

Lichen simplex chronicus and prurigo

Other dermatitis

Papulosquamous disorders

$13 \quad 2$

Psoriasis

Pityriasis rosea

Lichen planus

Other papulosquamous disorders

Urticaria and erythema

Urticaria

Radiation-related disorders of the skin and subcutaneous tissue

Other acute skin changes due to ultraviolet radiation

Skin changes due to chronic exposure of nonionizing radiation

Disorders of skin appendages

8

1

4

2

2

$56 \quad 12$

Nail disorders

Alopecia areata

Cicatricial alopecia (scarring hair loss)

Acne

9

16

15

2

5

9

54

45

Rosacea

Follicular cysts of the skin and subcutaneous tissue

Other follicular disorders

Other disorders of the skin and subcutaneous tissue

Vitiligo

Other disorders of pigmentation

Seborrheic keratosis

1

7

1

5

13

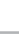

1

1

1

0

0

\begin{tabular}{l}
12 \\
4 \\
\hline
\end{tabular}

3

2
0




\begin{tabular}{|c|c|c|}
\hline Diagnosis & In person & E-consult \\
\hline Other disorders of the skin and subcutaneous tissue & 57 & 10 \\
\hline Atrophic disorders of the skin & 5 & 1 \\
\hline Hypertrophic disorders of skin & 5 & 0 \\
\hline Granulomatous disorders of skin and subcutaneous tissue & 4 & 1 \\
\hline Vasculitis limited to skin & 2 & 0 \\
\hline Ulcer of lower limb, not otherwise classified & 2 & 0 \\
\hline Other disorders of skin and subcutaneous tissue, not elsewhere classified & 5 & 1 \\
\hline Multiple superficial injuries & 1 & 0 \\
\hline Arthropod bite & 1 & 0 \\
\hline $\begin{array}{l}\text { Diseases of veins, lymphatic vessels and lymph nodes, not elsewhere } \\
\text { classified }\end{array}$ & 9 & 0 \\
\hline Varicose veins of lower extremities with inflammation (stasis dermatitis) & 9 & 0 \\
\hline Disorders of the oral cavity, salivary glands, and jaws & 3 & 2 \\
\hline Other specified disorders of gingiva and edentulous alveolar ridge & 1 & 0 \\
\hline Mucocele of salivary gland & 1 & 1 \\
\hline Diseases of lips & 0 & 1 \\
\hline Diseases of the tongue & 1 & 0 \\
\hline Congenital malformations, deformations and chromosomal abnormalities & 3 & 2 \\
\hline Other congenital malformations of the mouth & 3 & 0 \\
\hline Other congenital malformations of skin & 0 & 1 \\
\hline Neurofibromatosis & 0 & 1 \\
\hline Mycoses & 27 & 3 \\
\hline Dermatophytosis & 10 & 2 \\
\hline Other superficial mycoses & 17 & 1 \\
\hline Viral infections characterized by skin and mucous membrane lesions & 12 & 1 \\
\hline Viral warts & 12 & 1 \\
\hline Pediculosis, acariasis, and other infestations & 1 & 0 \\
\hline Scabies & 1 & 0 \\
\hline Other viral diseases & 2 & 0 \\
\hline Viral infection, unspecified & 2 & 0 \\
\hline Benign neoplasms & 17 & 4 \\
\hline Benign lipomatous neoplasm & 3 & 0 \\
\hline Hemangioma and lymphangioma, any site & 3 & 0 \\
\hline Melanocytic naevi of trunk & 6 & 2 \\
\hline Other benign neoplasms of skin & 5 & 2 \\
\hline Melanoma and other malignant neoplasms of the skin & 9 & 2 \\
\hline Other malignant neoplasms of skin & 9 & 2 \\
\hline Metabolic disorders & 2 & 0 \\
\hline Disorders of lipoprotein metabolism and other lipidemias & 2 & 0 \\
\hline Total & 374 & 77 \\
\hline
\end{tabular}

grouped dermatologic diagnoses, other studies have also found that dermatitis and eczema, ${ }^{1,5-7}$ disorders of the pilosebaceous follicle or acne, ${ }^{1,3,5-7}$ psoriasis, ${ }^{1,4}$ fungal diseases or dermatophyte infection, ${ }^{1-3,5}$ seborrheic dermatitis, ${ }^{1,3}$ and benign neoplasms and hyperplasias ${ }^{1}$ were among the most frequently diagnosed dermatologic diseases.

Although these diagnoses are common in the nonincarcerated population, some factors in the prison environment may 
contribute. Bayle and colleagues suggested that stress in the prison environment and smoking may contribute to the high frequency of disorders of the pilosebaceous unit. ${ }^{1}$ The smoking habits of our study population were not assessed. In addition, age, substance addiction and length of detention have been associated with dermatologic disease. ${ }^{5}$ Finally, some studies have suggested that personal hygiene and overcrowding may play a role. ${ }^{2,3,5,7}$ Brauner and Goodheart highlighted some of the potential difficulties in executing dermatologic treatments for prisoners, including limitations to the dosing frequency of pills and the dispensing of topical compounds, or the use of harsh soaps and skin care products. ${ }^{7}$ Furthermore, in the Eastern Ontario correctional facilities, there is a limited formulary of available products. ${ }^{10}$

The concept that e-consult is an effective tool to provide care to vulnerable populations is not novel. ${ }^{11}$ Coates and colleagues reviewed the accuracy and reliability of e-consult (teledermatology) and suggested that outcomes were comparable to in-person encounters. ${ }^{12}$ Our study supports the use of e-consult for the management of skin disease in prisoners. The most common dermatologic conditions treated in our study would likely be amenable to e-consult, thereby decreasing costs and increasing security - prisoners not housed at Collins Bay Institution were required to travel with escorts to Collins Bay for in-person consults, incurring travel costs and raising safety concerns with the transport of prisoners outside of their institutions. In addition, diagnosis and treatment might be provided in a timelier manner with e-consult, particularly if there are a limited number of inperson consultations per year. Thus, e-consult might provide an alternative to service an unmet medical need for this vulnerable population.

\section{Limitations}

Our study evaluates exclusively male inmates from Eastern Ontario, many of whom came from a single institution. Although these parameters may limit the generalizability of our study results, the findings are in keeping with published data, suggesting that our results are in keeping with a global pattern; however, further investigations are required.

A high proportion of included cases were prisoners at Collins Bay Institution, which may indicate a referral bias. Because of concerns with inmate transport and security, as well as the complexity of communication between institutions, the "in-house" inmates at Collins Bay Institution may have been more likely to receive an in-person dermatologic consult than inmates from other institutions. Our study design did not allow us to evaluate unmet need.

Only inmates referred for dermatologic consultation were included, therefore the sample was not randomized, and the incidence or prevalence of dermatologic disease in the incarcerated population cannot be calculated. In addition, any skin diseases treated successfully by other physicians, such as the prison physician, would not have been included, which would bias our study's results to capture more complex or treatmentresistant skin diseases.

\section{Conclusion}

The skin diseases that affected this federally incarcerated population in Ontario were common skin diseases, which is in agreement with other studies. Future studies investigating both male and female prisoners across Canada may capture more generalizable data for Canadian prisoners. To evaluate the incidence of dermatologic diseases in this vulnerable population, a random sample of all inmates could be evaluated for skin disease, instead of narrowing the study population to only those patients referred to a dermatologist.

The results of our chart review suggest that e-consult may be used to service the incarcerated population when in-person encounters are not possible. Future studies investigating the effectiveness of e-consult in prisons across Canada would strengthen this observation.

\section{References}

1. Bayle P, Cuzin L, Paul C, et al. Prisoners and skin diseases in Toulouse, France: epidemiological analysis and evaluation of life impact. 7 Eur Acad Dermatol Venereol 2009;23:52-7.

2. Oninla OA, Onayemi O. Report: Skin infections and infestations in prison inmates. Int 7 Dermatol 2012;51:178-81.

3. Oninla OA, Onayemi O, Olasode OA, et al. Pattern of dermatoses among inmates of Ilesha prison Nigeria. Niger Postgrad Med f 2013;20:174-80.

4. Coury C, Kelly B. Prison dermatology: experience in the Texas Department of Criminal Justice dermatology clinic. 7 Correct Health Care 2012;18:302-8.

5. Mannocci A, Di Thiene D, Semyonov L, et al. A cross-sectional study on dermatological diseases among male prisoners in southern Lazio, Italy. Int 7 Dermatol 2014;53:586-92.

6. Kocaturk E, Kocaturk A, Kayala M. Prevalence of skin diseases in female prisoners in Turkey: analysis of impact of prison conditions and psychological stress. Acta Dermatovenerol Croat 2014;22:26-31.

7. Brauner GJ, Goodheart HP. Dermatologic care behind bars. 7 Am Acad Dermatol 1988;18:1066-73.

8. Photographs of federal penitentiaries. Ottawa: Parole Board of Canada. Available: http://pbc-clcc.gc.ca/victims/photos/Ontario/kingston_1-eng.shtml (accessed 2015 June 9).

9. Ontario region. Ottawa: Correctional Service Canada. Available: www.csc-scc. gc.ca/institutions/001002-3000-eng.shtml (accessed 2015 June 9).

10. Audit of medication management. Ottawa: Correctional Service Canada. Available: www.csc-scc.gc.ca/publications/005007-2520-eng.shtml\#_TocI (accessed 2016 June 6).

11. James WD. The use of technology in providing dermatologic care to vulnerable populations. Cutis 2012;89:53-4.

12. Coates SJ, Kvedar J, Granstein RD. Teledermatology: from historical perspective to emerging techniques of the modern era. Part I: history, rationale, and current practice. 7 Am Acad Dermatol 2015;72:563-74.

Affiliations: Division of Dermatology (Gavigan, McEvoy, Walker), Department of Medicine, University of Ottawa; Division of Dermatology (Gavigan, McEvoy, Walker), Department of Medicine, Ottawa Hospital Research Institute, Ottawa, Ont.

Contributors: All of the authors were involved in the study design, the acquisition or analysis of the data, and the drafting and revising of the manuscript. All of the authors approved the final version to be published and agreed to act as guarantors of the work.

Acknowledgements: The authors would like to thank Dr. Tim Ramsay for assisting with the methodology of this study, and Dr. Jeff Turnbull for reviewing the manuscript.

Supplemental information: For reviewer comments and the original submission of this manuscript, please see www.cmajopen.ca/content/4/2/ E326/suppl/DC1 